\title{
Design, modelling and testing of electro-optical transmitters for the central pixel of the MAGIC telescope camera.
}

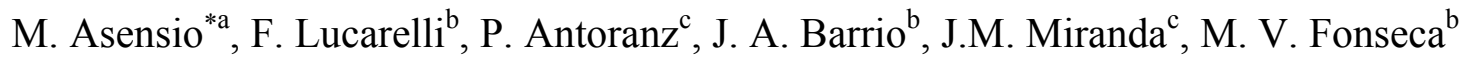 \\ ${ }^{a}$ Dept. Infra., I. Sistemas Aeroespaciales y Aerop., Universidad Politécnica de Madrid \\ ${ }^{\mathrm{b}}$ Dept. de Física Atómica, Molecular y Nuclear Universidad Complutense de Madrid \\ ${ }^{c}$ Dept. de Física Aplicada III, Universidad Complutense de Madrid
}

\begin{abstract}
In this work we have built an electro-optical system for the transmission of low frequency analogue signals through optical fibre. The main goal was to achieve minimum pulse distortion with maximum dynamic range. The system has been used in the framework of the MAGIC telescope experiment for the transmission of the analogue output from a photo-multiplier dedicated to optical observation of astrophysical objects, in particular pulsars. The received signal polarizes an infrared LED $(\lambda=850 \mathrm{~nm})$, which converts the pulse into an optical analogue pulse. The electro-optical pulse is transmitted by means of a multi-mode optic fibre and finally amplified and filtered by the optical receiver. The whole system has been tested using a pulse generator resembling the type of pulsed signal we expect from pulsars, that is with period of about tens of milli-seconds and few milli-seconds wide. The system was calibrated in order to: a) obtain a fixed relation between the received pulse and the final data and b) enhance the dynamic range and low distortion. In what follows, we show the behaviour of the optical transmitter under different pulse shapes, amplitude and frequencies up to several hundred Hz. The electro-optical system has been mounted on the MAGIC telescope and tested successfully with the observation of the pulsed optical signal from the Crab pulsar.
\end{abstract}

Keywords: Transmitter, Receiver, photodiode, LED, optical fibre, photomultiplier (PMT), Crab Pulsar, fast analog signal transfer.

\section{INTRODUCTION}

The MAGIC telescope ${ }^{1}$ is an innovative detector to measure very high-energy gamma radiation from astrophysical sources. The telescope detects the very short flashes of atmospheric Čerenkov radiation produced in the interaction of the cosmic gamma-rays with the atmospheric nuclei. A shower of relativistic charged secondary particles develop deep in the atmosphere and produce a cone of Cerenkov radiation as their charged particles move downward faster than the speed of light in the air ${ }^{2}$.

The main and unique characteristic of the telescope is the huge diameter of its mirror, $17 \mathrm{~m}$. That, along with a system of signal transmission based on optical fibre and digital sampling of signal with $300 \mathrm{MHz}$ Flash ADCs, allows for an extremely low energy threshold of primary gamma-rays of $30 \mathrm{GeV}$.

The main detector, or camera, is located at the focus of the telescope and consists of a matrix of 577 fast-response photomultipliers (PMTs) ${ }^{3}$, which register the very fast Čerenkov pulses (of 5-20 ns duration) emitted in the atmospheric cascades produced by the cosmic gamma-rays. The camera, shown in Fig. 1, has a diameter of $1.5 \mathrm{~m}$, and is composed by an inner ring of 397 1" PMTs (type ET9116B) and an outer ring of 180 1.5" PMTs (type ET9117B). All PMTs have a quantum efficiency of the $25-30 \%{ }^{4,5}$. In a second phase of upgrading of the telescope, it is foreseen to use new hybrid light detectors with a quantum efficiency of $45 \%{ }^{6}$.

Imaging Atmospheric Čerenkov Detectors (telescopes) ${ }^{7}$ can be used to detect the optical emission of an astronomical object by measuring the slow variations in the DC current of the camera PMTs. This technique was already applied by former Cerenkov telescopes which detected the pulsed optical emission from the Crab pulsar and estimated the photon

*mariano.asensio@upm.es; phone 03491336 6322; fax 034913366633 
content of the nebula surrounding the pulsar ${ }^{7}$. The centre of the MAGIC camera was especially designed to host a dedicated PMT for optical observations, the so called central pixel or central PMT. Such modified PMT was installed at the end of March '05 and tested successfully with the observation of the optical pulsed emission from the Crab pulsar.

In what follows, we describe the electro-optical transmission system of the MAGIC central PMT (type EMI9116). The DC output signal from the base of the PMT is transmitted through optical fibre to the counting house, where it is converted and stored for off-line analysis. The development of this system is a fundamental part of the whole central PMT installation, which also included: design of a mechanical support for the central PMT; Electrical feed of the central PMT and the Transmitter; Electric and magnetic protections; Filtered, digitisation and storing of the signal received.

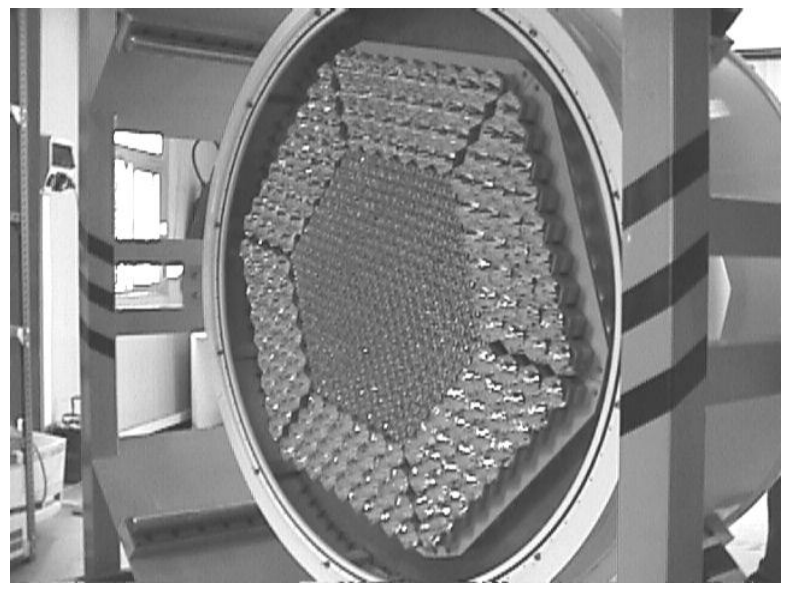

Figure 1: MAGIC telescope camera.

\section{DESIGN}

\section{Constrains}

Some limitations were imposed to us for the development of our transmission system since the telescope has been working since 2001. The signal transmission from the MAGIC Telescope camera is made through optical fibres which have the following specifications:

$$
\begin{aligned}
& \text { Optical Fibre } \\
& \circ \text { Longitude } \mathrm{L}=170 \mathrm{~m} \text {. } \\
& \circ \text { Graded index fibre multimode with } 50 / 125 \mu \mathrm{m} \text { core } / \text { cladding, } \lambda=850 \mathrm{~nm} \text {. }
\end{aligned}
$$

- Diamond E 2000 connector.

The Diamond connectors were chosen over other systems because of its integrated safety clips, which interrupts the light beam when disconnecting the fibre.

The optical transmitters installed for the rest of the photomultipliers use an electronic circuit with filter's bandwidth out of the range of the slow optical signals we want to detect and make use of VCSEL Laser Emitters, more suitable to follow the fast Čerenkov light pulses emitted in the atmospheric showers. We decided, instead, to use cheaper and more robust devices to transmit the signal. We expect from the Crab pulsar a pulsed signal of frequency $\cong 30 \mathrm{~Hz}$, pulse width of $\cong 3 \mathrm{~ms}$ and amplitude which depends on the PMT gain and the pre-amplifier at the PMT base. Therefore, we decided to use high speed LEDs.

We made two prototypes, the first with Agilent technology (HFBR-1412 TX 0329, HFBR-2416 RX 0347), and the second with Honeywell technology. Both have been working perfectly in laboratory, but the first option needed two STE 2000 adapters, one for the transmitter and another one for the receiver. Thus, once the system was installed in the 
telescope in order to be tested, we found an attenuation of about $50 \%$ due to the adaptors. Therefore, we decided to install the second one, from Honeywell.

\section{Transmitter System}

The used transmitter is an E-2000 packaged Honeywell High power LED, HFE 4050-014. It is a high radiance GaAlAs LED optimized for coupling into small fibre core diameters.

- $\quad$ Fiber Coupled Power, Poc $=70 \mu \mathrm{W}\left(\mathrm{I}_{\mathrm{F}}=100 \mathrm{~mA}, \mathrm{NA}=0.20, \mathrm{~T}=25^{\circ}, 50 / 125 \mu \mathrm{m}\right)^{9}$.

- Peak wavelength, $\lambda \mathrm{p}=850 \mathrm{~nm}$. Bandwidth (full-width half maximum FWHM) $=50 \mathrm{~nm}$.

- Analog bandwidth: $85 \mathrm{MHz}$ (high); rise time $\mathrm{t}_{\mathrm{R}}=6 \mathrm{~ns}$, fall time $\mathrm{t}_{\mathrm{F}}=6 \mathrm{~ns}$.

- As the current varies (typically from $10 \mathrm{~mA}$ to $100 \mathrm{~mA}$ ), the light intensity increases proportionally.

- Power launched into the Fiber optic cable is linear with the forward current of the LED between $20 \mathrm{~mA}$ and $50 \mathrm{~mA}$.

Figure 2 shows the measured I-V plot of the LED. The LED's linear model used for the simulations with PSpice ${ }^{\circledR}$ is obtained from this plot.

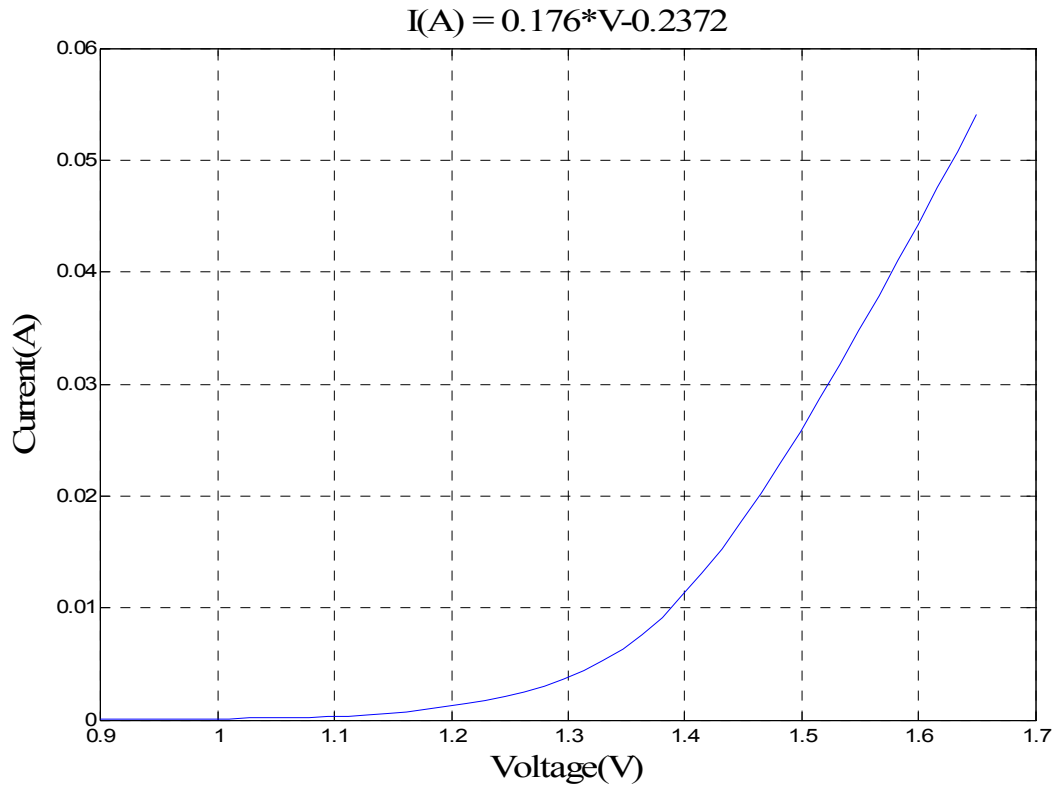

Figure 2: I V characteristic plot and linear model of the HFE 4050-014 LED.

Linear model: $f(x)=p 1 * x+p 2$

Coefficients (with $95 \%$ confidence bounds):

$$
\begin{aligned}
& \mathrm{p} 1=0.176(0.1707,0.1812) ; \mathrm{r}=5.68 \Omega(5.86,5.52) \\
& \mathrm{p} 2=-0.2372(-0.2452,-0.2292) ; \mathrm{V}_{\gamma}=1.35 \mathrm{~V}(1.26,1.44)
\end{aligned}
$$

Goodness of fit: SSE: 6.037e-006 R-square: 0.9976 Adjusted R-square: 0.9974 RMSE: 0.0006814

The photomultiplier comes with a pre-amplifier integrated in its base. So, the transmitter's input signal is the output signal of this circuit. We expect that the signal at the input of the transmitter will consist of a DC signal plus a pulsed signal plus noise. We are only interested in the pulsed signal, so if we remove the DC signal, we expect to obtain a signal-to-noise ratio smaller than one.

Figure 3 shows the electronic circuit that processes the signal received from the base of the photomultiplier and converts it to an optical one through the LED, and how the LED is polarized. The amplifier's gain is chosen to maximize the transmitter's dynamic range. We chose the LED's operating point at 40mA. At this point, the LED works linearly and the optical signal has enough power to reach the receiver without attenuation. 
The transmitter is installed into the telescope's camera, where the room temperature varies between $25^{\circ} \mathrm{C}$ in winter and $40^{\circ} \mathrm{C}$ in summer.

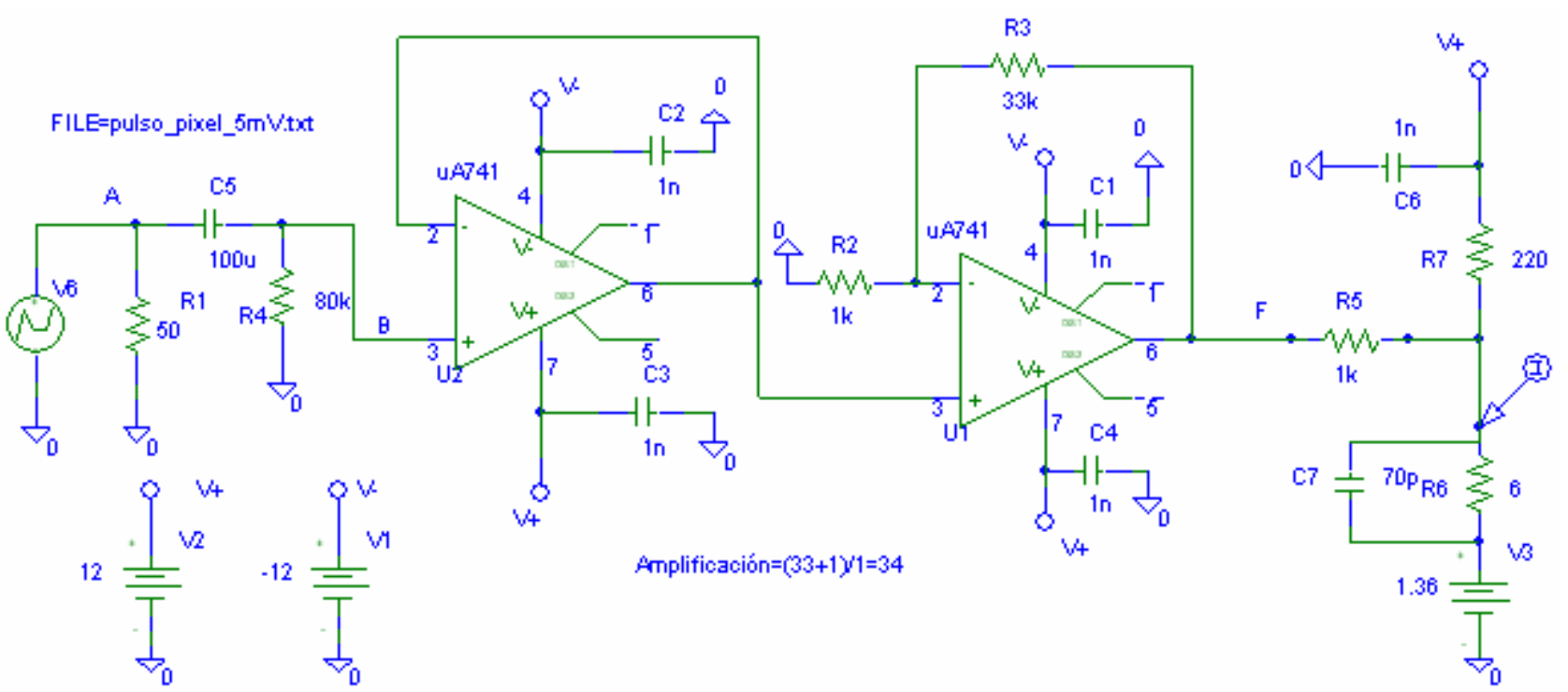

Figure 3: Light-emitting diode (LED) transmitter-design High-Speed configuration.

Figure 4 shows the transmitter's frequency response, taking as input signal the photomultiplier base's output signal and as output signal the current through the LED.

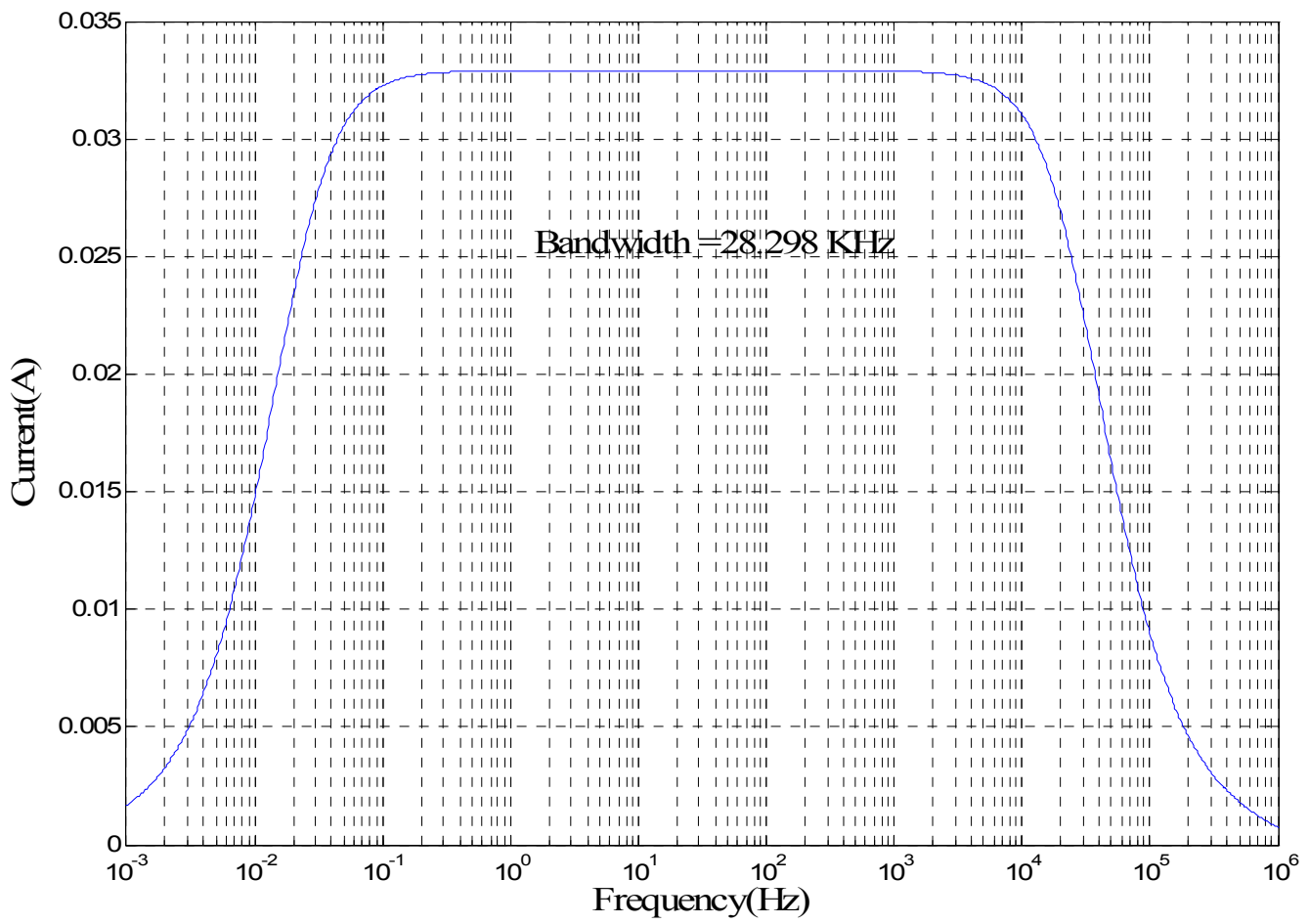

Figure 4: Transmitter System's Frequency Response. 


\section{Optical Fibre}

The model of optical fibre used in the MAGIC telescope was already installed before we made this work. The main characteristics of the installed fibre are:

- Fibre attenuation: $2.20 \mathrm{~dB} / \mathrm{km}(0.4 \mathrm{~dB}$ in $170 \mathrm{~m})$.

- Insertion loss (IL): $-0.07 \mathrm{~dB}$.

- Minimum RMS pulse spreading $\sigma_{\mathrm{m}} \approx 14 \mathrm{ps} / \mathrm{Km}(2.38 \mathrm{ps}$ in $170 \mathrm{~m})$.

- Empirical bandwidth: $\mathrm{BW} \approx 12.857 \mathrm{GHz} \cdot \mathrm{km}$.

We expect that, for such fiber length, the coupling and transmission losses, and optical pulse dispersion are very small.

\section{Photodiode Receiver}

As for optical receiver, we are using the Honeywell HFD3038-002, a $25 \mathrm{MHz}$ silicon PIN plus pre-amplified analog receiver for high speed operation ${ }^{10}$. The receiver is placed in the counting house (containing the rest of the MAGIC Telescope data acquisition electronics), in a conditioned room where the temperature varies between $15^{\circ} \mathrm{C}$ in winter and $30^{\circ} \mathrm{C}$ in summer.

Its technical specifications report a typical rise and fall times of $3 \mathrm{~ns}$, a responsivity $(\mathrm{R})$ of $7.5 \mathrm{mV} / \mu \mathrm{W}$ and a maximum input power of $150 \mu \mathrm{W}^{10}$.

Figure 5 shows the electronic circuit made to process the photodiode's output signal.

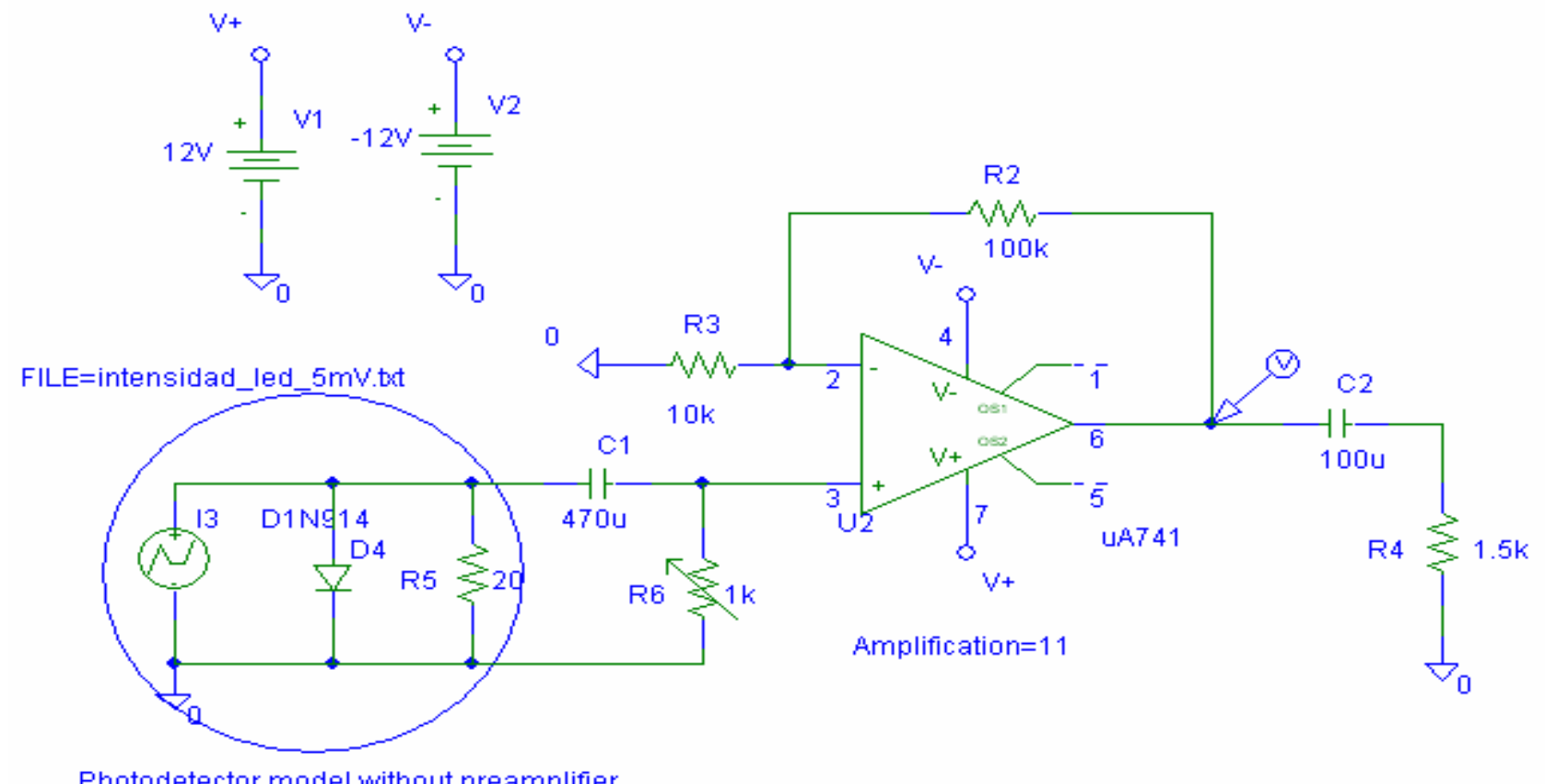

Figure 5: Circuit diagram of the optical receiver.

\section{SIMULATION}

\section{Simulation of the transmitter}

We can simulate the effect of the white noise we expect in the transmitter (see below), by using a random sequence with a correlation time much smaller than the shortest time constant of the system. The Band-Limited White Noise Simulink ${ }^{\circledR}$ block produces such a sequence. The correlation time of the noise is the sample rate of the block. For accurate simulations, we use a correlation time much smaller than the fastest dynamics of the system. We can get good results by specifying

$$
\mathrm{t}_{\mathrm{c}} \approx \frac{1}{100} \frac{2 \pi}{\mathrm{f}_{\max }}
$$

where $\mathrm{f}_{\max }$ is the bandwidth of the system in $\mathrm{rad} / \mathrm{sec}$. 
The parameters that define the Band-Limited White Noise Simulink ${ }^{\circledR}$ block are:

Noise power: The height of the power spectral density (PSD) of the white noise.

Sample time: The correlation time of the noise.

The output signal of the Simulink block (Figure 6: Scope1) is saved into a file *.txt. We can import, from PSpice, the file generated with Simulink writing the file *.txt in the field FILE of the VPWL_FILE source (Figure 3).

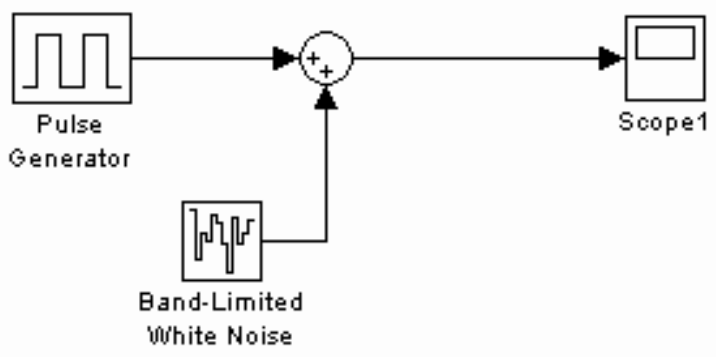

Figure 6: Simulink circuit to simulate the pulsed signal plus noise.

\subsection{Transmitter Simulation Results}

The fundamental idea of this paragraph is to simulate the output signal of the MAGIC central PMT that we expect when the telescope is pointing to the Crab pulsar. We expect a very weak input signal for the transmitter, with a lot of noise. We only can simulate this kind of signals. To do the simulation we generate a pulsed signal of amplitude $5 \mathrm{mV}$, whose characteristics has already been described, plus a white noise with a height of the power spectral density (PSD) equal to $2.5 \mathrm{e}-10 \mathrm{~W} / \mathrm{Hz}$. Taking into account the transmitter's bandwidth, we have generated a signal with a signal-to-noise ratio $=$ $-48 \mathrm{~dB}$. The signal shown in Figure 7 simulates the voltage signal delivered by the PMT base. Figure 3 shows how this voltage signal is processed. The current signal that passes through the LED is showed in Figure 7.
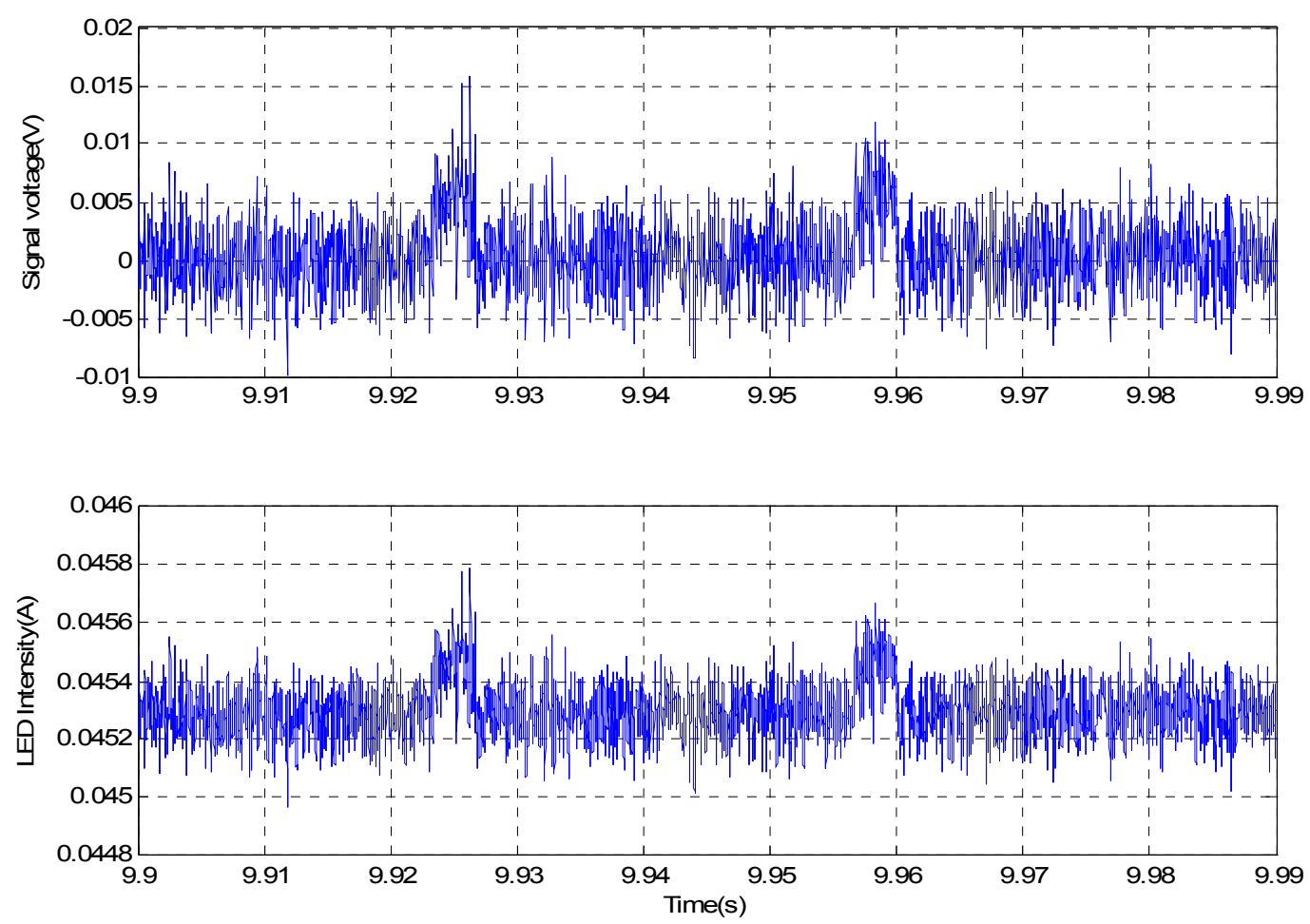

Figure 7: Transmitter's input and output Signals. 
Polarization applied to the LED allows that the LED current to remain into the linear range with the optical power ${ }^{8}$.

\section{Simulation of the receiver}

The electronic circuit (Figure 5) that processes the voltage signal delivered by the photodiode consists of a first stage which filters the DC level, a second amplification stage, and a last filtering stage when we can choose the low and high cutoff frequency.

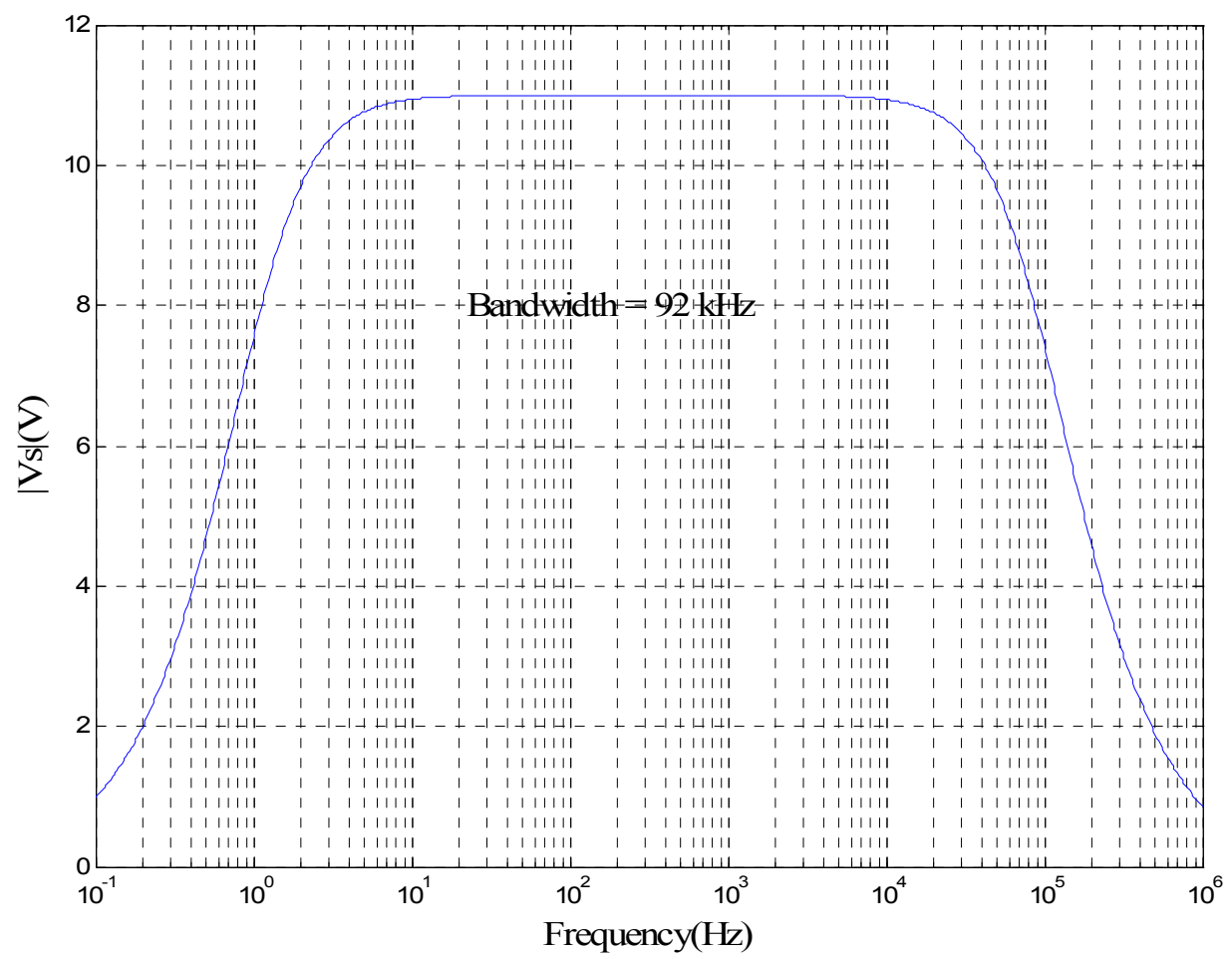

Figure 8: Receiver System's Frequency Response.

Figure 5 shows a photodiode's simplified model without preamplifier stage and a low-pass filter in order to choose the low cutoff frequency. The bandwidth of this circuit is showed in Figure 8.

We can obtain the generated photodiode current $\left(\mathrm{i}_{\mathrm{p}}\right)$ from the linear relation:

$$
i_{p}=r \cdot P_{d}
$$

where $\mathrm{r}$ is the photodiode responsivity $\left(\mathrm{r} \approx 0.6 \mathrm{~A} / \mathrm{W}\right.$, theoretical value) and $\mathrm{P}_{\mathrm{d}}$ is the optical absorbed power by the detector. We can estimate $P_{d}$ from coupled power into the fibre by the LED. Simulation without preamplifier stage is possible if we fill the field FILE of the current source (Figure 5) with the data obtained $i_{p}$.

\subsection{Receiver simulation Results}

Once the whole system was installed in the telescope, we made several tests. One of this tests consisted of proving the relationship between the transmitter voltage input signal and photo-detector receiver (photodiode plus preamplifier stage) voltage output signal. This characteristic is showed in Figure 9:

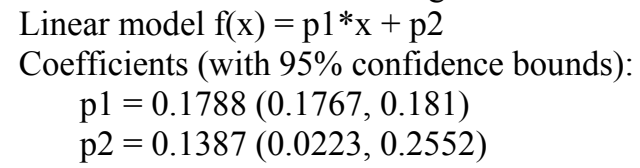

Goodness of fit: SSE: 0.06303, R-square: 0.9998, Adjusted R-square: 0.9998, RMSE: 0.08876

To obtain the photo-detector receiver voltage output signal when the amplitude of the transmitter voltage input signal is $5 \mathrm{mV}$, we can use the linear fitting: $\mathrm{f}(5 \mathrm{mV})=0.1788 * 5 \mathrm{mV}+0.1387=1.0327 \mathrm{mV}$. 


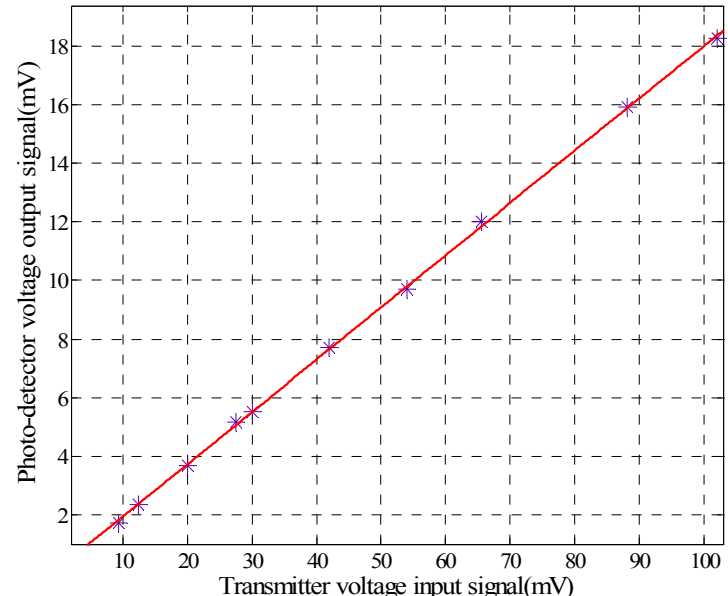

Figure 9: Relation transmitter input photo-detector output.

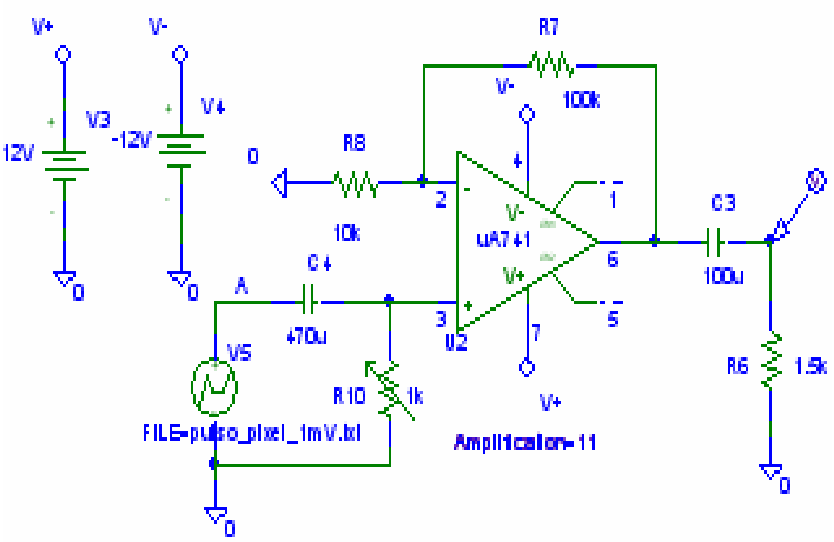

Figure 10: Receiver circuit used in simulation.

The photo-detector receiver voltage output signal consists of a DC level $(-2.3 \mathrm{~V})$, plus a pulsed signal (amplitude $1 \mathrm{mV}$ ) plus noise. To simulate the receiver system, we use the circuit showed in Figure 10, filling the field FILE of the source with the photo-detector voltage output signal. Figure 11 shows the results of the simulation.
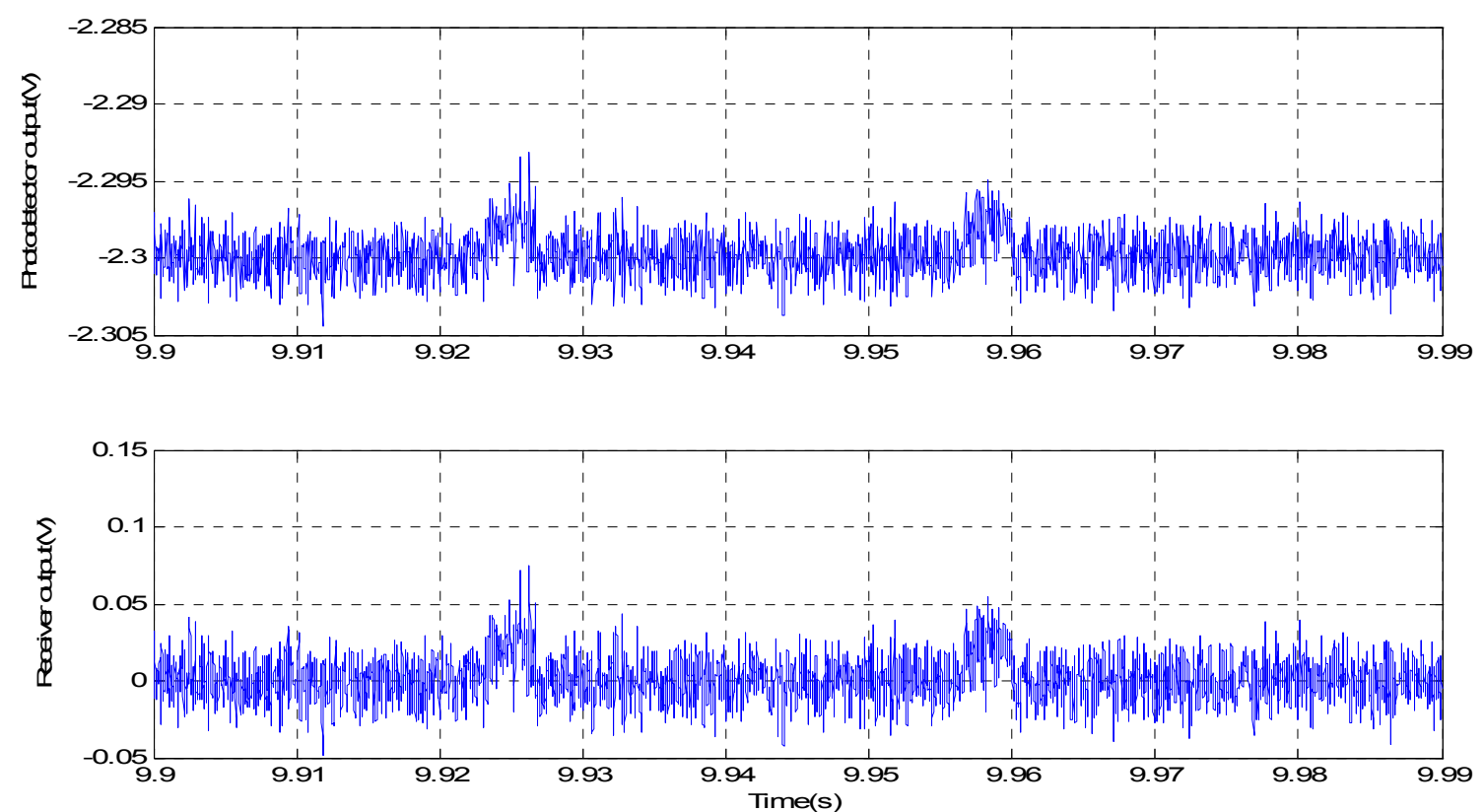

Figure 11: Photo-detector's output signal and receiver's output signal.

\section{ON-SITE TESTS AND RESULTS}

\section{On-site Tests and Results}

In order to verify that the installation was correct, we transmitted a series of pulsed signals from the camera of the telescope to the counting house by feeding them directly into the transmitter by means of a pulse generator. Figure 12 
shows the two test signals at the receiver output. These two output signals correspond to two transmitter input signals of amplitude $10 \mathrm{mV}$ and $100 \mathrm{~m}$, respectively.
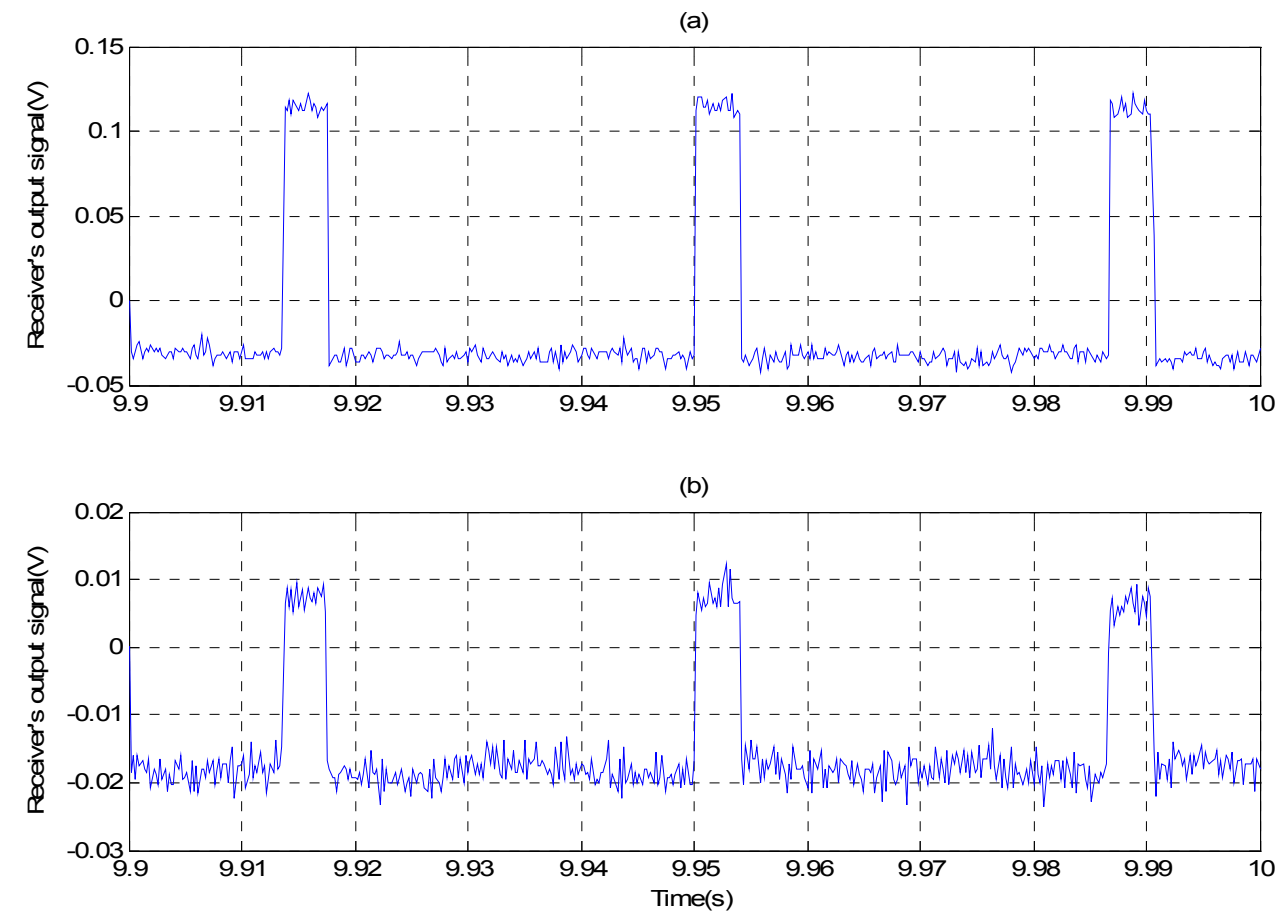

Figure 12: Results of the on-site tests (170m of optical fibre): receiver output for (a) a $100 \mathrm{mV}$ input pulse, (b) a $10 \mathrm{mV}$ input pulse.

After these successful on-site tests, we set the MAGIC Telescope to observe the optical emission from the Crab pulsar. The signal registered by the central PMT, and transmitted through the electro-optical system described above, was digitalised during 20 minutes using a National Instruments PCI-NI 6034-E ADC card, at a $2 \mathrm{kHz}$ sampling rate. This digitalised signal was folded with the Crab pulsar frequency $(\sim 29.78 \mathrm{~Hz})$ in order to detect the Crab optical pulsed emission. Figure 13 shows the so-called lightcurve of the Crab pulsar, which represents the variation of the light intensity within a pulsar rotation period, which is in agreement with the expected one. In fact, two narrow pulses around 3 ms wide $\left(1 / 10\right.$ of a Crab period) clearly appear in the light curve as we expected ${ }^{1}$. This result shows the good performance of the electro-optical system installed both in amplitude and time response, allowing the whole central pixel set-up to measure DC variations as small as $0.2 \%$, as it is estimated from the lower peak of the lightcurve.

\footnotetext{
${ }^{1}$ The appearance of these two peaks with different amplitudes and shifted by half a period is related to the orientation of the axis rotation of the pulsar with respect to the observer ${ }^{7}$.
} 


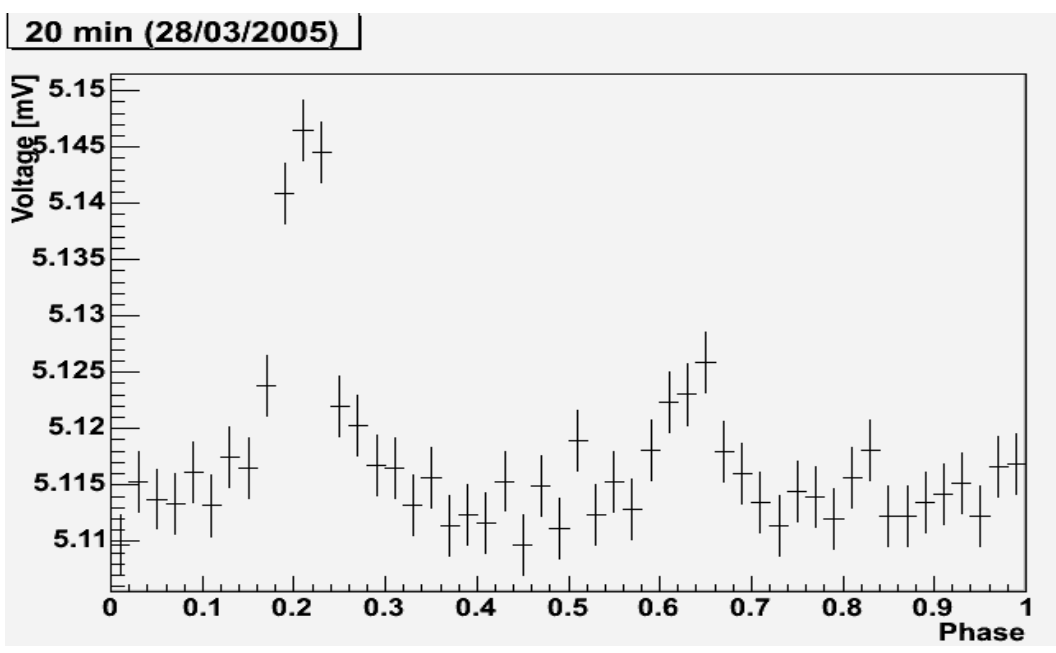

Figure 13: Measured Crab pulsar lightcurve. In this figure, the $\mathrm{x}$-axis represents a period of the pulsar rotation, while $y$-axis the mean voltage measured by the ADC card, which is proportional to the light intensity measured by the central PMT and transmitted through the optical fibre.

\section{CONCLUSIONS}

The MAGIC Čerenkov telescope is located at the Roque de los Muchachos observatory, on the island of La Palma (Canary Islands, Spain). In this work, we have presented the design and implementation of a system to transmit the signal collected by the so called central PMT of the MAGIC telescope camera, a photomultiplier operating in continuous mode. The signal of the photomultiplier is transmitted analogically over an optic fibre from the camera to the counting house, located $170 \mathrm{~m}$. away, where it is digitalised and stored for later analysis.

Optic fibre transmission over these distances respects the shape of pulses, which is not the case for coaxial cables. Time response and linearity are well inside the needed requirements.

Tests performed in laboratory conditions using pulse generators allowed us to check the behaviour of the transmitter-receptor device. Finally the whole system has been installed in the telescoped and used to observe the pulsed signal from the pulsar inside the Crab Nebula. Its light curve, obtained after a careful analysis of the data recorded, proves the viability of this approach. This result shows the good performance of the electro-optical system installed, both in amplitude and time response, allowing the whole central pixel set-up to measure DC variations as small as $0.2 \%$.

The simultaneous observation of optical and gamma radiation with the MAGIC telescope, using its Čerenkov camera and the system described above may open new perspectives in the field of High Energy Astrophysics.

\section{ACKNOWLEDEGMENTS}

The authors thank the financial support given by the CICYT (project FPA2003-9543-C02-01) to make this work. We also acknowledge Eckart Lorenz, Razmik Mirzoyan, Manel Martínez and the IFAE engineering team, our colleagues at the High Energy Physics Group of the U.C.M. and the Department of Infrastructure, Aerospace Systems and Airports of the U.P.M. for their valuable help.

\section{REFERENCES}

1. M. V. Fonseca, The MAGIC telescope project, Acta Physica Polonica B, vol. 30, pp. 2331 (1999).

2. T.K. Gaisser, Cosmic Rays and Particle Physics, Cambridge University Press, 1990.

3. W.R. Leo, Techniques for Nuclear and Particle Physics Experiments, Springer-Verlag, Second Edition.

4. A. Ostankov, D. Paneque et al., A study of the new hemispherical 6-dynodes PMT from electron tubes, Nuclear Instruments and Measurements in Physics Research A, vol. 442, Issue 1-3, pp. 117-123 (2000) 
5. J. Gebauer et al., Evaluation of a New High QE Photomultiplier for Air Čerenkov Telescopes, Nuclear Instruments and Measurements in Physics Research A 518 (2004) 615

6. E. Lorenz et al., Progress on the Development of a high QE, red extended Hybrid Photomultiplier for the 2nd Phase of the MAGIC telescope, Nuclear Instruments and Measurements in Physics Research A 504 (2003) 280-285

7. E. Oña-Wilhelmi, J. Cortina, O.C. de Jager, V. Fonseca. Determination of the Night Sky Background around the Crab pulsar using its optical pulsation. Astroparticle Physics Vol. 22:95-102, 2004.

8. E. Lorenz, et al. A fast, large dynamic range analog signal transfer system based on optical fibers, Nuclear Instruments and Measurements in Physics Research A 461 (2001) 517.

9. Honeywell, HFD4050-014 Datasheet.

10. Honeywell, HFD3038 Datasheet. 